\title{
Management of Atrial Fibrillation in COVID-19 Pandemic
}

\author{
Yu-Feng Hu${ }^{1}$, Wen-Han Cheng ${ }^{2}$, Yuan Hung ${ }^{3}$, Wen-Yu Lin ${ }^{4}$, Tze-Fan Chao ${ }^{2}$, Jonan Liao ${ }^{5}$, \\ Yenn-Jiang $\operatorname{Lin}^{2}$, Wei-Shiang $\operatorname{Lin}^{4}$, and Shih-Ann Chen ${ }^{2}$ \\ ${ }^{1}$ Taipei Veteran General Hospital \\ ${ }^{2}$ Taipei Veterans General Hospital \\ ${ }^{3}$ Tri-Service General Hospital \\ ${ }^{4}$ Tri-Service General Hospital, National Defense Medical Center \\ ${ }^{5}$ Taipei Venterans General Hospital
}

May 26, 2020

\begin{abstract}
The health crisis due to coronavirus disease 2019 (COVID-19) shocks the world with more than million infections and casualties. COVID-19 could present from mild illness to multi-organ involvement, especially acute respiratory distress syndrome. Cardiac injury and arrhythmias including atrial fibrillation (AF) are not uncommon in COVID-19. COVID-19 is highly contagious, and the therapy against the virus remains premature and largely unknown. These make the management of AF patients during pandemic particularly challenging. We here describe possible pathophysiology link between COVID-19 and AF, and therapeutic considerations for AF patients during this pandemic.
\end{abstract}

\section{Introduction}

The global pandemic of coronavirus disease 2019 (COVID-19) has caused more than million infections and hundred thousand deaths. ${ }^{1}$ As of May 18th, 2020, there were more than 4,500,000 patients infected globally with over 300,000 fatalities. The disease is caused by the severe acute respiratory coronavirus 2 (SARS-CoV2), a single-stranded RNA virus belonging to the family Coronaviridae. ${ }^{2}$ Although the majority of COVID-19 patients $(81 \%)$ presented with mild illness, more than $15 \%$ of the patients developed severe diseases to multiorgan decompensation. ${ }^{3}$ These critical patients requiring intensive care were usually older and had underlying comorbidities, e.g., coronary artery disease, hypertension, diabetes and cerebrovascular disease. ${ }^{4}$ The severe complications included acute respiratory distress syndrome (ARDS) (29\%), acute cardiac injury (12\%), and secondary infection (10\%). ${ }^{5}$ COVID-19 patients with cardiac injury had a higher incidence of malignant arrhythmias and mortality rate than those without. ${ }^{6}$ The incidence of arrhythmias varies in different studies, ranging from $5.9 \%$ to $16.7 \%$. ${ }^{4,6}$

Atrial fibrillation (AF) is the most common arrhythmias associated with aging and a variety of cardiovascular comorbidities. It is not be surprising that the new-onset or pre-existing AF could be frequently observed in COVID-19 patients. The Italian Ministry of Health reported concurrent AF in 19-22\% of COVID-19 patients. ${ }^{7,8}$ In the latest pharmacological trial using remdesivir in 53 hospitalized COVID-19 patients, 3 patients developed new-onset $\mathrm{AF}(6 \%) .^{9}$

The management of AF is complex and includes rhythm and rate control, and prevention of stroke. The COVID-19 pandemic has brought unprecedented and significant challenges in the management of AF patients with COVID-19. Therefore, we review the evidence of COVID-19 and AF from relevant pathophysiology to clinical management, solicit pivotal issues, and provide provisional recommendations.

The pathophysiology link between COVID-19 and AF 


\section{The role of angiotensin-converting enzyme 2}

Angiotensin-converting enzyme 2 (ACE2) converts angiotensin I (Ang I) and angiotensin II (Ang II) into the biologically active peptide Ang-(1-9) and Ang-(1-7), respectively. ${ }^{10,11}$ This provides counter-regulatory effects of ACE in the renin-angiotensin-aldosterone system (RAAS). ACE2 could be a functional receptor and cellular entry point for SARS-CoV-2 and SARS-CoV to invade target cells in heart, lung, and kidney where prominent expressions of ACE2 are observed. ${ }^{12,13}$ Although the current evidence remains insufficient, SARS-CoV-2 might cause direct injury of myocardium by binding to ACE2. ${ }^{14}$ Previous data from SARS$\mathrm{CoV}$ might provide some theoretical mechanism for COVID-19 because SARS-CoV and SARS-CoV-2 have similar affinity to ACE2. ${ }^{12,13}$ The downregulation of myocardial ACE2 expressions after SARS-CoV infection leads to excessive accumulation of Ang II and causes myocardial injury, remodeling and even adverse cardiac outcomes. ${ }^{15}$ This might underlie AF arrhythmogenesis in COVID-19. Atrial ACE2 expressions would decrease the levels of transforming growth factor- $\beta 1$ (TGF- $\beta 1$ ) and collagen deposition. ${ }^{16}$ ACE2 might be able to regulate cardiac action potential. ${ }^{17}$ Laboratory data also demonstrated that ACE2 metabolites, Ang-(1-7), could modulate electrophysiological characteristics and calcium hemostasis in atrial tissue and pulmonary vein cardiomyocytes. ${ }^{18,19}$ The downregulation of ACE2 in COVID-19 might increase AF vulnerability and its perpetuation.

\section{Cytokine storms and $A F$}

SARS-CoV-2 triggers immune responses with a cytokine storm syndrome. ${ }^{20}$ The majority of severe cases had elevated levels of infection-related biomarkers and inflammatory cytokines, such as tumor necrosis factor alpha (TNF- $\alpha$ ), IL-6, interleukin $1 \beta$ (IL-1 $\beta$ ), interleukin-2 (IL-2), IL-7, IL-10, granulocyte colony-stimulating factor, granulocyte macrophage colony-stimulating factor, interferon- $\gamma$ induced protein, monocyte chemoattractant protein 1, fibroblast growth factor, vascular endothelial growth factor, and macrophage inflammatory protein $1-\alpha \cdot{ }^{5,14,20}$ Cytokine storm might trigger a violent immune system response, and cause ARDS and hypoxemia, which indirectly damage myocardial cells. ${ }^{5,14,20,21}$ Additionally, some cytokines have direct effects on cardiomyocytes. The aforementioned factorsmight induce atrial electrical and structural remodeling, and lead to AF occurrence.

Although the electrophysiological effects of cytokines remain incompletely understood, distinctive cytokines have been shown to induce $\mathrm{AF}$ in bench work and translational researches. ${ }^{22,23} \mathrm{TNF}-\alpha$ increases $\mathrm{AF}$ vulnerability and exerts direct effects on atrial structural and electrical remodeling. ${ }^{24,25} \mathrm{TNF}-\alpha$ induced triggered activity in pulmonary vein cardiomyocytes, which was attributed to increased activity of sodium/calcium exchanger (NCX) and impaired sarcoplasmic reticulum (SR) ATPase. ${ }^{24}$ Abnormal Ca ${ }^{2+}$ leak from SR could also be induced by TNF- $\alpha .{ }^{26} \mathrm{TNF}-\alpha$ and IL-1 $\beta$ both impair cardiac contractility, ${ }^{25,26,27}$ and contribute to arrhythmogenesis through mechano-electrical feedback. IL-6 reduces cardiac connexins and promotes electrical remodeling during acute inflammatory status. ${ }^{28}$ Different cytokines, including TNF- $\alpha$, IL-1 $\beta$, fibroblast growth factor, and vascular endothelial growth factor all increase cardiac fibrosis and underlie arrhythmogeneity. ${ }^{29,30}$ The activation of inflammasome NLRP3-related signals was hypothesized after SARS-CoV-2 infection. ${ }^{31}$ Increased activity of NLRP3 inflammasome was similarly observed in AF patients, which might be associated with macrophage recruitment, fibrosis, and myocardial dysfunction in the heart. ${ }^{32,33}$ Several therapeutic trials have been launched according to these inflammatory hypotheses. Hydroxychloroquine (HCLQ), a potential therapeutic candidate for COVID-19 could reduce activity of NLRP3 inflammasome in inflammatory cells. ${ }^{34}$ The immune-suppressive agent, tocilizumab (an IL-6 inhibitor), was approved for phase 3 trial in severely ill COVID-19 patients in order to reduce the complication of COVID-19 by the US Food and Drug Administration.

\section{Drug interactions between AF medications and proposed COVID-19 treatment}

Many investigational drugs have been proposed for COVID-19. Safety alerts due to drug side effects including long QT interval and ventricular arrhythmias were raised for HCLQ and azithromycin. ${ }^{35}$ This red flag should be emphasized more while these drugs were prescribed along with AF medications for rate and rhythm control. AF medications include $\beta$-blockers, non-dihydropyridines (DHP) calcium channel blockers (CCB), 
digoxin and antiarrhythmic agents. ${ }^{36}$ The use of these medications increases the risk for serious side effects such as bradycardia, heart failure, long QT interval, or torsades de pointes. Therefore, the guideline of medical treatment for AF has been provided by the international consensus. ${ }^{36}$ Drug-drug interaction between AF and COVID-19 medications might share common metabolism and side effects (Table 1). In addition to the evaluation of baseline renal function, liver function and ECG parameters when initiating medications, a thoughtful consideration of drug interactions, adjustment, and monitoring is critical for patient safety and therapeutic efficiency.

\section{Medications for rate control}

Most $\beta$-blockers can be safely used with investigational COVID-19 medications. Verapamil is P-glycoprotein (P-gp) related and metabolized by CYP3A4-type cytochrome P450-dependent enzymes (Table 2). Since HCLQ exerts impact on hyperpolarization-activated current $\mathrm{I}_{\mathrm{f}}$ modulation in sinoatrial node and atrioventricular node cells, co-administration of verapamil and HCLQ may cause bradycardia and conduction disturbance. ${ }^{37}$ Digoxin is well-known for its narrow therapeutic range ${ }^{38}$ Moreover, investigational COVID-19 medications which consist of substrate of CYP3A4 and P-gp, such as HCLQ, may potentially increase digoxin level and its toxicity, resulting in conduction disturbance. ${ }^{39}$ Therefore, ECG monitoring for bradycardia and conduction disturbance should be considered.

\section{Antiarrhythmic agents}

HCLQ, a derivative of chloroquine, is notoriously known for drug-induced QT prolongation. ${ }^{35,40,41,42}$ Moreover, azithromycin also induces QT prolongation. ${ }^{35}$ The administration of antiarrhythmic agents, including amiodarone, dronedarone, flecainide and propafenone with HCLQ or azithromycin may further increase the risk of QT prolongation and induce ventricular tachyarrhythmia or sudden cardiac death (Table 1). Therefore, ECG monitoring for the QT interval is important. Drugs should be either withheld or removed if QT interval is longer than $500 \mathrm{~ms}$ as generally recommended. The increased plasma level of antiarrhythmic agents while being used with these antiviral drugs would potentially increase the risk of malignant arrhythmias and sudden cardiac death.

In addition to ventricular tachyarrhythmia, co-administration of antiarrhythmic agents and investigational COVID-19 medications could increase the blood concentration of class Ic and class III antiarrhythmic drugs, and expose the patients to risk of atrial proarrhythmic events. The increased blood concentration of class Ic and III antiarrhythmic agents, including propafenone and amiodarone, would change conduction properties of the atrial tissue. For example, persistent atrial flutter may occur with the increase of atrial refractory period. ${ }^{43,44,45}$

\section{Hypercoagulable state}

COVID-19 is associated with a hypercoagulable status. ${ }^{46,47}$ COVID-19 patients with acute respiratory failure presented a severe hypercoagulability rather than consumptive coagulopathy. Fibrin formation and polymerization may predispose to thrombosis and correlate with a worse outcome. ${ }^{46}$ The incidence of venous thromboembolism could be as high as $25 \%$ in COVID-19 patients with severe pneumonia. ${ }^{48}$ In a different cohort, the composite outcome of symptomatic acute pulmonary embolism, deep-vein thrombosis, ischemic stroke, myocardial infarction, or systemic arterial embolism was $31 \% \cdot{ }^{49}$ The stroke rate in COVID patients was around $1-2 \% \cdot{ }^{4,50}$ The incidence of stroke is not as high as venous thromboembolism. The evidence is still needed to establish a causal relationship between stroke and COVID-19. The International Society on Thrombosis and Haemosthasis (ISTH) and American Society of Hematology (ASH) recommends prophylactic low molecular weight heparin for venous thromboembolism in all hospitalized COVID-19 patients in the absence of any contraindications ${ }^{51}$ However, supporting evidence ofprophylactic anticoagulants for stroke in AF patients with COVID-19remains sparse. We do not know whether the anticoagulants in COVID-19 patients with AF should be treated more aggressively even under low CHA2DS2-VASc score (0 for male and 1 for female). Hypercoagulable state in severe COVID-19 diseases was frequently observed with concurrent multi-organ failure, which would also significantly increase the risk of bleeding. 


\section{Interactions between oral anticoagulants and therapies of COVID-19}

Stroke prevention with oral anticoagulants (OACs), including vitamin K antagonist (warfarin) and nonvitamin $\mathrm{K}$ antagonist OACs (NOACs), is the cornerstone for the managements of AF. Before initiations of OACs for AF patients infected with COVID-19, it is necessary to check platelet counts, coagulation profiles (e.g. prothrombin time and activated partial thromboplastin time), renal s and liver functions which are probably affected with severe infections. Importantly, these parameters should be monitored and followed up closely during the disease course, and the dosages of OACs should be adjusted when indicated.

\section{Warfarin}

It is known that warfarin has multiple drug-drug or drug-food interactions. However, there were no significant interactions between warfarin and remdesivir or HCLQ (Table 1). For patients initiating warfarin, international normalized ratio (INR) should be monitored every day until the therapeutic range (INR 2-3) is achieved. Thereafter, INR could be followed up when clinically indicated.

\section{NOACs}

NOACs can directly inhibit thrombin (dabigatran) or factor Xa (rivaroxaban, apixaban and edoxaban) and are prescribed more commonly for stroke prevention in AF. ${ }^{52}$ NOACs have been recommended as the firstline medications unless contraindications are present. ${ }^{36}$ Different from warfarin, NOACs have less drug-drug or drug-food interactions and are prescribed at a fixed dose following individual dosage criteria without routine monitorof the drug concentrations or anticoagulation activities. ${ }^{53}$ However, NOACs still have drugdrug interactions with some medications. An important interaction mechanism for all NOACs is significant gastrointestinal re-secretion over a P-gp transporter after absorption in the gut. Competitive inhibition of this pathway will result in increased plasma levels. ${ }^{53}$ Besides, CYP3A4-dependent elimination is relevantly involved in the hepatic clearance of rivaroxaban and apixaban. Strong CYP3A4 inhibition or induction may affect plasma concentrations. ${ }^{53}$ Based on these concepts, COVID-19 medications which could compete or inhibit P-gp or CYP3A4 would result in increased plasma levels of NOACs.

As Table 2 shows, HCLQ is a substrate of CYP3A4, and therefore, may pontentially increase the plasma concentrations of NOACs. Generally, NOACs could be co-administered with HCLQ with either usual dosages (apixaban or rivaroxaban) or dosage adjustments (dabigatran or edoxaban). However, marcolides (e.x. erythromycin or clarithromycin or azithromycin) are moderate P-gp competitors and strong CYP3A4 inhibitors, and may increase the exposures of NOACs by $15-20 \%$ for dabigatran, $60 \%$ for apixaban, $90 \%$ for edoxaban and $34 \%$ (erythromycin) $/ 54 \%$ (clarithromycin) for rivaroxaban. ${ }^{53}$ Therefore, if HCLQ plus azithromycin regimen is adopted to AF patients with COVID-19, the NOACs should be co-administered with cautions.

Remdesivir, a nucleotide analogue prodrug that inhibits viral RNA polymerases, provides a great hope for the treatment of COVID-19 infection. ${ }^{9}$ Remdesivir is expected to have no significant effects on the metabolisms or eliminations of all four NOACs, and could be co-administrated with NOACs.

Future directions

Although the routine monitorings of the plasma concentrations of NOACs and the anti-factor Xa activities are generally not necessary in the daily practice, the plasma concentrations of NOACs haven been shown significantly associated with bleeding and ischemic events. ${ }^{54,55}$ If the measurements of plama concentrations of NOACs were available in the institutions caring the COVID-19 infected patients, the monitoring of plasma levels of NOACs may be considered when drug-drug interactions between NOACs and medications for COVID-19 are concerned. More clinical experiences are necessary for the concomitant use of OACs and COVID-19 therapies.

\section{Catheter ablation of AF}

Urgent or elective ablation of AF

Catheter ablation for AF patients with active infection is generally contraindicated, which is similarly applied 
to COVID-19 patients. ${ }^{56}$ Medical treatment to control ventricular rate or rhythm with adequate anticoagulation based on the risk score will be the preferred managment. ${ }^{56}$ Electrical or chemical cardioversion is a reasonable alternative for refractory arrhythmias. COVID-19 fears put interventional laboratories on lockdown. The asymptomatic carriers or mildly symptomatic COVID-19 patients remain highly contagious and could be unexpectedly admitted. During active pandemic, the hospitalization of these patient would significantly increase the risk for group infection within hospitals. As most AF ablation is not an urgent procedure, these procedures are suggested to be postponed for several weeks or months during active pandemic to avoid unexpected outbreak in hospitals. ${ }^{57}$ However, some electrophysiology (EP) procedures which are considered urgent or emergent for the diseases with substantially risk of clinical decompensation, hospitalization, or death might not be avoidable. These include AF, atrial flutter, or atrioventricular nodal ablation which is hemodynamically significant, severely symptomatic, drug and/or cardioversion refractory, or preexcited AF with syncope or cardiac arrest. ${ }^{57}$

\section{Electrical cardioversion for AF in acute heart failure}

$\mathrm{AF}$ could induce heart failure due to the loss of atrial systole, and irregular and/or rapid ventricular conduction. ${ }^{58}$ Therefore, electrical cardioversion to restore sinus rhythm might improve heart failure. However, in COVID-19 patients, acute heart failure is highly attributed to cytokine storm, instead of AF induced hemodynamic changes. AF and heart failure are bothharmful consequence of cytoine storm. Electrical cardioversion might contribute to a minor role, and instead, immunological treatment might be associated with recovery of left ventricular systolic function. ${ }^{59}$ The procedure of electrical cardioversion might increase the risk to healthcare workers. For examples, the delivery of aerosol generating procedures is needed to a patient infected during general anesthesia. ${ }^{60}$ The potential complications from electrical cardioversion including ventricular fibrillation due to unsynchronized shock, heart block, bradycardia, transient myocardial dysfunction, and hypotension would lead to unavoidable urgent resuscitation. ${ }^{60}$ All of these increase infection risk for caregivers. AF recurrence after successful cardioversion is not uncommon in the patients with heart failure. ${ }^{58}$ Therefore, we suggested medical treatment should be maximized including adequate rate control, anticoagulation, or diuresis or inotropic support. Prompt electrical cardioversion should be avoided.

\section{General practice after COVID-19 pandemic}

Excessive delays could threaten patient prognosis even for catheter ablations on the regular basis to palliate drug-refractory arrhythmias. Therefore, the re-initiation of regular procedure with adequate protection and infection control is recommended after thepandemic is under control. The setup of hospital-based infection control protocols for ablation procedures will be critical to avoid outbreak in the hospital. ${ }^{61}$ The procedure and preparation for AF ablation are still mostly complied with current guideline ${ }^{56}$ However, several recommendations are made for the preventing viral transmission as adequacy. There is no consensus whether universal screening should be applied for all the patients before interventional procedures. However, the test for COVID-19 should be performed, provided that recent travel history, contact history, fever after high-risk exposures, or typical symptoms are highly suggesting COVID-19 infection. All the admitted patients and their companions should declare their health condition, travel, and contact history. On-site visitors are not avoided, and the stay in the hospital for patients and visitors should be minimized to decrease the risk of nosocomial infection. Instead, virtual video visit through cell phone or webcam is suggested. A dictated catheter laboratory with independent air-supply and ventilation, or negative pressure should be reserved for suspicious COVID-19 cases. ${ }^{57}$ Split operation is strictly implemented in our country. All staff is divided into 2-3 independent working shifts without personal contact during the daily activities. High flow oxygen, intubation, noninvasive positive pressure ventilation, or transesophageal echocardiography are generally not recommended as these increase the chance of virus spreading and transmission to the operators. In addition to personal protective equipment of medical personnel, patients should also wear face masks during hospital stay and procedures. CT scanning or intracardiac echocardiography could be considered to exclude atrial thrombus rather than transesophageal echocardiography before AF ablation procedure. Either the procedure time or hospital stay should be minimized. ${ }^{57}$

Practice for confirmed or suspicious COVID-19 patients 
The recommendations for the pre/post AF procedure and catheter laboratory and caring units can be similarly applied from the peri-operative recommendations. ${ }^{62}$ The patients with confirmed or suspected COVID19 infection should be scheduled as the last case of the day in the dictated laboratory and followed by extensive sterilization of environment or instruments. To minimize the transport of infected patients, elective intubation with highly selective patients in the intensive care unit or in a negative ventilation pressure room should be performed prior to entering the EP laboratory. ${ }^{57}$

\section{Follow-up after catheter ablation}

Current guideline suggests all patients who undergo catheter ablation for AF should be followed up at a minimum of 3 months following the ablation procedure, and then every 6 months for at least 2 years. A personal visit with 12-lead ECG and more intense monitoring is also recommended. However, the strategy of follow-up during or after COVID-19 pandemic is prone to change.${ }^{56}$ For examples, in-person clinic visit is recommended to be avoided, and instead, tele-health/virtual visits could be adopted to minimize unnecessary exposure. ${ }^{57}$ The inspections of incision site after device implantation or catheter ablation can be managed via tele-health utilizing a video conference or mobile photography. Instead of conventional monitoring device such as 24-hour Holter Monitoring, remote monitor of heart rhythm via mobile device is suggested to decrease on-site visits.

\section{References}

1. CucinottaD, VanelliM: WHO declares COVID-19 a pandemic. Acta Biomed. Mattioli 1885, 2020, pp. $157-160$.

2. ZhouP, YangXLou, WangXG, et al.: A pneumonia outbreak associated with a new coronavirus of probable bat origin. Nature Nature Research, 2020;579:270-273.

3. WuZ, McGooganJM: Characteristics of and Important Lessons from the Coronavirus Disease 2019 (COVID-19) Outbreak in China: Summary of a Report of 72314 Cases from the Chinese Center for Disease Control and Prevention. JAMA - J. Am. Med. Assoc. American Medical Association, 2020, pp. 1239-1242.

4. WangD, HuB, HuC, et al.: Clinical Characteristics of 138 Hospitalized Patients with 2019 Novel Coronavirus-Infected Pneumonia in Wuhan, China. JAMA - J Am Med Assoc American Medical Association, 2020;323:1061-1069.

5. HuangC, WangY, LiX, et al.: Clinical features of patients infected with 2019 novel coronavirus in Wuhan, China. Lancet Lancet Publishing Group, 2020;395:497-506.

6. GuoT, FanY, ChenM, WuX, ZhangL, HeT, WangH, WanJ, WangX, LuZ: Cardiovascular Implications of Fatal Outcomes of Patients with Coronavirus Disease 2019 (COVID-19). JAMA Cardiol American Medical Association, 2020;

7. Report sulle caratteristiche dei pazienti deceduti positivi a COVID-19 in Italia Il presente report è basato sui dati aggiornati al 20 Marzo 2020.

8. Characteristics and Outcomes of Patients Hospitalized for COVID-19 and Cardiac Disease in Northern Italy - PubMed [Internet]. [cited 2020 May 20],. Available from: https://pubmed.ncbi.nlm.nih.gov/32383763/?from_single_result=32383763\&expanded_search_query=32383763

9. GreinJ, OhmagariN, ShinD, et al.: Compassionate Use of Remdesivir for Patients with Severe Covid-19. N Engl J Med Massachusetts Medical Society, 2020;.

10. FerrarioCM, JessupJ, ChappellMC, AverillDB, BrosnihanKB, TallantEA, DizDI, GallagherPE: Effect of angiotensin-converting enzyme inhibition and angiotensin II receptor blockers on cardiac angiotensinconverting enzyme 2. Circulation 2005;111:2605-2610.

11. DonoghueM, HsiehF, BaronasE, GodboutK, GosselinM, StaglianoN, DonovanM, WoolfB, RobisonK, JeyaseelanR, BreitbartRE, ActonS: A novel angiotensin-converting enzyme-related carboxypeptidase (ACE2) 
converts angiotensin I to angiotensin 1-9. Circ Res 2000;87.

12. LetkoM, MarziA, MunsterV: Functional assessment of cell entry and receptor usage for SARS-CoV-2 and other lineage B betacoronaviruses. Nat Microbiol Nature Research, 2020;5:562-569.

13. WallsAC, ParkYJ, TortoriciMA, WallA, McGuireAT, VeeslerD: Structure, Function, and Antigenicity of the SARS-CoV-2 Spike Glycoprotein. Cell Cell Press, 2020;181:281-292.e6.

14. ZhengYY, MaYT, ZhangJY, XieX: COVID-19 and the cardiovascular system. Nat. Rev. Cardiol. Nature Research, 2020, pp. 259-260.

15. OuditGY, KassiriZ, JiangC, LiuPP, PoutanenSM, PenningerJM, ButanyJ: SARS-coronavirus modulation of myocardial ACE2 expression and inflammation in patients with SARS. Eur J Clin Invest 2009;39:618-625.

16. ZhouT, WangZ, FanJ, ChenS, TanZ, YangH, YinY: Angiotensin-converting enzyme-2 overexpression improves atrial remodeling and function in a canine model of atrial fibrillation. J Am Heart Assoc 2015;4:e001530.

17. Activation of angiotensin-converting enzyme 2 improves cardiac electrical changes in ventricular repolarization in streptozotocin-induced hyperglyc. . - PubMed - NCBI [Internet]. [cited 2020 May 19],. Available from: https://www.ncbi.nlm.nih.gov/pubmed/?term $=24741027$

18. LuYY, WuWS, LinYK, ChengCC, ChenYC, ChenSA, ChenYJ: Angiotensin 1-7 modulates electrophysiological characteristics and calcium homoeostasis in pulmonary veins cardiomyocytes via MAS/PI3K/eNOS signalling pathway. Eur J Clin Invest Blackwell Publishing Ltd, 2018;48.

19. ChenSA, ChangMS, ChiangBN, ChengKK, LinCI: Electromechanical effects of angiotensin in human atrial tissues. J Mol Cell Cardiol 1991;23:483-493.

20. MehtaP, McAuleyDF, BrownM, SanchezE, TattersallRS, MansonJJ: COVID-19: consider cytokine storm syndromes and immunosuppression. Lancet. Lancet Publishing Group, 2020, pp. 1033-1034.

21. QinC, ZhouL, HuZ, ZhangS, YangS, TaoY, XieC, MaK, ShangK, WangW, TianDS: Dysregulation of immune response in patients with COVID-19 in Wuhan, China. Clin Infect Dis NLM (Medline), 2020;.

22. GuoY, LipGYH, ApostolakisS: Inflammation in atrial fibrillation. J. Am. Coll. Cardiol. Elsevier USA, 2012, pp. 2263-2270.

23. HuYF, ChenYJ, LinYJ, ChenSA: Inflammation and the pathogenesis of atrial fibrillation. Nat. Rev. Cardiol. Nature Publishing Group, 2015, pp. 230-243.

24. LeeSH, ChenYC, ChenYJ, ChangSL, TaiCT, WongcharoenW, YehHI, LinCI, ChenSA: Tumor necrosis factor- $\alpha$ alters calcium handling and increases arrhythmogenesis of pulmonary vein cardiomyocytes. Life Sci 2007;80:1806-1815.

25. Aschar-SobbiR, IzaddoustdarF, KorogyiAS, et al.: Increased atrial arrhythmia susceptibility induced by intense endurance exercise in mice requires TNF $\alpha$. Nat Commun Nature Publishing Group, 2015;6.

26. DuncanDJ, YangZ, HopkinsPM, SteeleDS, HarrisonSM: TNF- $\alpha$ and IL-1 $\beta$ increase Ca2+ leak from the sarcoplasmic reticulum and susceptibility to arrhythmia in rat ventricular myocytes. Cell Calcium Elsevier Ltd, 2010;47:378-386.

27. PrabhuSD: Cytokine-induced modulation of cardiac function. Circ. Res. 2004, pp. 1140-1153.

28. LazzeriniPE, Laghi-PasiniF, AcampaM, et al.: Systemic Inflammation Rapidly Induces Reversible Atrial Electrical Remodeling: The Role of Interleukin-6-Mediated Changes in Connexin Expression. J Am Heart Assoc American Heart Association Inc., 2019;8.

29. BorthwickLA, WynnTA, FisherAJ: Cytokine mediated tissue fibrosis. Biochim. Biophys. Acta - Mol. Basis Dis. 2013, pp. 1049-1060. 
30. ChungCC, LinYK, ChenYC, KaoYH, LeeTI, ChenYJ: Vascular endothelial growth factor enhances profibrotic activities through modulation of calcium homeostasis in human atrial fibroblasts. Lab Investig Springer Nature, 2020;100:285-296.

31. Castaño-RodriguezC, HonrubiaJM, Gutiérrez-ÁlvarezJ, et al.: Role of severe acute respiratory syndrome coronavirus viroporins E, 3a, and 8a in replication and pathogenesis. MBio American Society for Microbiology, 2018;9.

32. SuetomiT, WillefordA, BrandCS, ChoY, RossRS, MiyamotoS, BrownJH: Inflammation and NLRP3 Inflammasome Activation Initiated in Response to Pressure Overload by Ca2+/Calmodulin-Dependent Protein Kinase II $\delta$ Signaling in Cardiomyocytes Are Essential for Adverse Cardiac Remodeling. Circulation Lippincott Williams and Wilkins, 2018;138:2530-2544.

33. YaoC, VelevaT, ScottL, et al.: Enhanced Cardiomyocyte NLRP3 Inflammasome Signaling Promotes Atrial Fibrillation. Circulation Lippincott Williams and Wilkins, 2018;138:2227-2242.

34. TangTT, LvLL, PanMM, WenY, WangB, LiZL, WuM, WangFM, CrowleySD, LiuBC: Hydroxychloroquine attenuates renal ischemia/reperfusion injury by inhibiting cathepsin mediated NLRP3 inflammasome activation. Cell Death Dis Nature Publishing Group, 2018;9.

35. SappJL, AlqarawiW, MacIntyreCJ, TadrosR, SteinbergC, RobertsJD, LaksmanZ, HealeyJS, KrahnAD: Guidance On Minimizing Risk of Drug-Induced Ventricular Arrhythmia During Treatment of COVID-19: A Statement from the Canadian Heart Rhythm Society. Can J Cardiol Elsevier BV, 2020;.

36. JanuaryCT, WannLS, CalkinsH, et al.: 2019 AHA/ACC/HRS Focused Update of the 2014 AHA/ACC/HRS Guideline for the Management of Patients With Atrial Fibrillation: A Report of the American College of Cardiology/American Heart Association Task Force on Clinical Practice Guidelines and the Heart Rhythm Society in Collaboration With the Society of Thoracic Surgeons. Circulation NLM (Medline), 2019;140:e125-e151.

37. CapelRA, HerringN, KallaM, YavariA, MiramsGR, DouglasG, BubG, ChannonK, PatersonDJ, TerrarDA, BurtonRAB: Hydroxychloroquine reduces heart rate by modulating the hyperpolarization-activated current If: Novel electrophysiological insights and therapeutic potential. Hear Rhythm Elsevier B.V., 2015;12:2186-2194.

38. BotelhoAFM, PierezanF, Soto-BlancoB, MeloMM: A review of cardiac glycosides: Structure, toxicokinetics, clinical signs, diagnosis and antineoplastic potential. Toxicon. Elsevier Ltd, 2019, pp. 63-68.

39. WesslerJD, GripLT, MendellJ, GiuglianoRP: The P-glycoprotein transport system and cardiovascular drugs. J. Am. Coll. Cardiol. 2013, pp. 2495-2502.

40. Drug interactions with antimalarial medications in older travelers: a clinical guide. - PubMed - NCBI [Internet]. [cited 2020 May 19],. Available from: https://www.ncbi.nlm.nih.gov/pubmed/?term=31776555

41. Structural bases for the different anti-fibrillatory effects of chloroquine and quinidine. - PubMed - NCBI [Internet]. [cited 2020 May 19],. Available from: https://www.ncbi.nlm.nih.gov/pubmed/?term=21233253

42. Rodríguez-MenchacaAA, Navarro-PolancoRA, Ferrer-VilladaT, RuppJ, SachseFB, Tristani-FirouziM, Sánchez-ChapulaJA: The molecular basis of chloroquine block of the inward rectifier Kir2.1 channel. Proc Natl Acad Sci U S A National Academy of Sciences, 2008;105:1364-1368.

43. TaiCT, LinYK, LanFC, ChenHY, DingYA, ChangMS, ChenSA: Conduction Properties of the Crista Terminalis in Patients with Atrial Flutter Due to Amiodarone Therapy for Atrial Fibrillation. PACE Pacing Clin Electrophysiol 2003;26:2241-2246.

44. TaiCT, ChiangCE, LeeSH, ChenYJ, YuWC, FengAN, DingYA, ChangMS, ChenSA: Persistent atrial flutter in patients treated for atrial fibrillation with amiodarone and propafenone: Electrophysiologic charac- 
teristics, radiofrequency catheter ablation, and risk prediction. J Cardiovasc Electrophysiol Futura Publishing Company Inc., 1999;10:1180-1187.

45. TaiCT, ChenSA, FengAN, YuWC, ChenYJ, ChangMS: Electropharmacologic effects of class I and class III antiarrhythmia drugs on typical atrial flutter: Insights into the mechanism of termination. Circulation Lippincott Williams and Wilkins, 1998;97:1935-1945.

46. SpieziaL, BoscoloA, PolettoF, CerrutiL, TiberioI, CampelloE, NavalesiP, SimioniP: COVID-19Related Severe Hypercoagulability in Patients Admitted to Intensive Care Unit for Acute Respiratory Failure. Thromb Haemost [Internet] Thromb Haemost, 2020 [cited 2020 May 20];. Available from: http://www.ncbi.nlm.nih.gov/pubmed/32316063

47. VioliF, PastoriD, CangemiR, PignatelliP, LoffredoL: Hypercoagulation and Antithrombotic Treatment in Coronavirus 2019: A New Challenge. Thromb Haemost [Internet] Georg Thieme Verlag KG, 2020 [cited 2020 May 20];. Available from: http://www.thieme-connect.de/DOI/DOI?10.1055/s-0040-1710317

48. CuiS, ChenS, LiX, LiuS, WangF: Prevalence of venous thromboembolism in patients with severe novel coronavirus pneumonia. J Thromb Haemost Wiley, 2020;.

49. KlokFA, KruipMJHA, van derMeerNJM, ArbousMS, GommersDAMPJ, KantKM, KapteinFHJ, vanPaassenJ, StalsMAM, HuismanMV., EndemanH: Incidence of thrombotic complications in critically ill ICU patients with COVID-19. Thromb Res Elsevier Ltd, 2020;.

50. AhmadI, RathoreFA: Neurological manifestations and complications of COVID-19: A literature review. J Clin Neurosci [Internet] J Clin Neurosci, 2020 [cited 2020 May 20];: Available from: https://linkinghub.elsevier.com/retrieve/pii/S096758682031078X

51. COVID-19 and VTE-Anticoagulation - Hematology.org [Internet]. [cited 2020 May 20],. Available from: https://www.hematology.org/covid-19/covid-19-and-vte-anticoagulation

52. ChaoTF, ChiangCE, LinYJ, ChangSL, LoLW, HuYF, TuanTC, LiaoJN, ChungFP, ChenTJ, LipGYH, ChenSA: Evolving changes of the use of oral anticoagulants and outcomes in patients with newly diagnosed atrial fibrillation in Taiwan. Circulation. Lippincott Williams and Wilkins, 2018, pp. 1485-1487.

53. The 2018 European Heart Rhythm Association Practical Guide on the use of non-vitamin K antagonist oral anticoagulants in patients with atrial fibri. . - PubMed - NCBI [Internet]. [cited 2020 May 19],. Available from: https://www.ncbi.nlm.nih.gov/pubmed/?term=29562325

54. ReillyPA, LehrT, HaertterS, ConnollySJ, YusufS, EikelboomJW, EzekowitzMD, NehmizG, WangS, WallentinL: The effect of dabigatran plasma concentrations and patient characteristics on the frequency of ischemic stroke and major bleeding in atrial fibrillation patients: The RE-LY trial (Randomized Evaluation of Long-Term Anticoagulation Therapy). J Am Coll Cardiol 2014;63:321-328.

55. Clinical outcomes, edoxaban concentration, and anti-factor Xa activity of Asian patients with atrial fibrillation compared with non-Asians in the E. .. - PubMed - NCBI [Internet]. [cited 2020 May 19],. Available from: https://www.ncbi.nlm.nih.gov/pubmed/?term=30590425

56. CalkinsH, HindricksG, CappatoR, et al.: 2017 HRS/EHRA/ECAS/APHRS/SOLAECE expert consensus statement on catheter and surgical ablation of atrial fibrillation: Executive summary. J Arrhythmia Elsevier B.V., 2017;33:369-409.

57. LakkireddyDR, ChungMK, GopinathannairR, et al.: Guidance for Cardiac Electrophysiology During the Coronavirus (COVID-19) Pandemic from the Heart Rhythm Society COVID-19 Task Force; Electrophysiology Section of the American College of Cardiology; and the Electrocardiography and Arrhythmias Committee of the Council on Clinical Cardiology, American Heart Association. Hear Rhythm Elsevier BV, 2020;.

58. Atrial Fibrillation in Heart Failure: What Should We Do? - PubMed [Internet]. [cited 2020 May 
20],. Available from: https://pubmed.ncbi.nlm.nih.gov/26419625/?from_single_result=26419625\&expanded_search_query $=26419625$

59. BelhadjerZ, MéotM, BajolleF, et al.: Acute heart failure in multisystem inflammatory syndrome in children (MIS-C) in the context of global SARS-CoV-2 pandemic. Circulation [Internet] Circulation, 2020 [cited 2020 May 20];:CIRCULATIONAHA.120.048360. Available from: https://www.ahajournals.org/doi/10.1161/CIRCULATIONAHA.120.048360

60. PerkinsGD, MorleyPT, NolanJP, et al.: International Liaison Committee on Resuscitation: COVID-19 Consensus on Science, Treatment Recommendations and Task Force Insights. Resuscitation [Internet] Resuscitation, 2020 [cited 2020 May 20];151. Available from: http://www.ncbi.nlm.nih.gov/pubmed/32371027

61. DrigginE, MadhavanMV., BikdeliB, et al.: Cardiovascular Considerations for Patients, Health Care Workers, and Health Systems During the COVID-19 Pandemic. J. Am. Coll. Cardiol. Elsevier USA, 2020, pp. $2352-2371$.

62. GreenlandJR, MichelowMD, WangL, LondonMJ: COVID-19 Infection: Implications for Perioperative and Critical Care Physicians. Anesthesiology NLM (Medline), 2020;.

63. Liverpool COVID-19 Interactions [Internet]. [cited 2020 May 20],. Available from: https://www.covid19druginteractions.org/

Table 1. Interactions between medications for atrial fibrillation and COVID-19.

\begin{tabular}{|c|c|c|c|}
\hline & Remdesivir & Hydroxychloroquine & Azithromycin \\
\hline Rate control drugs & Rate control drugs & Rate control drugs & Rate control drugs \\
\hline$\beta$-blockers & $\beta$-blockers & $\beta$-blockers & $\beta$-blockers \\
\hline Atenolol & - & - & - \\
\hline Bisoprolol & - & - & - \\
\hline Metoprolol & - & - & - \\
\hline Propranolol & - & - & - \\
\hline $\mathrm{CCB}$ & $\mathrm{CCB}$ & $\mathrm{CCB}$ & \\
\hline Diltiazem & - & - & - \\
\hline Verapamil & - & & - \\
\hline Others & Others & Others & \\
\hline Digoxin & - & & - \\
\hline Rhythm control drugs & Rhythm control drugs & Rhythm control drugs & Rhythm control drugs \\
\hline Amiodarone & - & & \\
\hline Dronedarone & No available data & & \\
\hline Flecainide & - & & \\
\hline Propafenone & - & - & \\
\hline Oral anticoagulants & Oral anticoagulants & Oral anticoagulants & Oral anticoagulants \\
\hline Apixaban & - & & \\
\hline Dabigatran & - & & \\
\hline Edoxaban & - & & \\
\hline Rivaroxaban & - & & \\
\hline Warfarin & - & - & - \\
\hline
\end{tabular}

CCB: calcium channel blockers; COVID-19: coronavirus disease 2019

: Potential substantially increased exposure of the medications; these drugs should not be prescribed together.

: Potential moderately increased exposure of the medications; dosage adjustment or close monitoring may be required. 
: Potential mildly increased exposure of the medications; the interactions are weak

-: No significant effects

Data about drug-drug interactions presented in this table were adopted from the work by Liverpool Drug Interactions Group which could be found at the following website: https://www.covid19-druginteractions.org ${ }^{63}$

Table 2. Pharmacokinetics of medications for atrial fibrillation and COVID-19.

COVID-19: coronavirus disease 2019

Medications that are P-glycoprotein substrates or inhibitors Medications that are P-glycoprotein substrates or inhibitors

$\beta$-blockers

Calcium channel blockers

Antiarrhythmic agents

Oral anticoagulants

Others

COVID-19 drugs

Medications that are CYP3A4 substrates

Calcium channel blockers

Antiarrhythmic agents

Bisoprolol (substrate/inhibitor), Propranolol (substrate/inhil

Oral anticoagulants

Diltiazem (substrate/inhibitor), Verapamil (substrate/inhibit

Amiodarone (inhibitor), Dronedarone (inhibitor), Propafenor

COVID-19 drugs Apixaban (substrate), Dabigatran (substrate), Edoxaban (su Digoxin (substrate)

Azithromycin (substrate/inhibitor)

Medications that are CYP3A4 substrates

Diltiazem, Verapamil

Amiodarone

Apixaban, Rivaroxaban

Hydroxychloroquine, Azithromycin 